\title{
COPD
}

\section{Dreifach-Fixkombination für instabile Patienten}

_ Viele Patienten mit chronisch obstruktiver Lungenerkrankung (COPD) sind unter ihrer Medikation weiterhin symptomatisch und/oder weisen ein erhöhtes Exazerbationsrisko auf. Statt mit einer Monotherapie mit einem langwirksamen Muskarinantagonisten (LAMA), einer dualen Bronchodilatation mit einem langwirksamen $\beta_{2}$-Agonisten (LABA) oder einer Kombination aus LABA und einem inhalativen Kortikoid (ICS) kann diesen Patienten mit einer Tripeltherapie aus LAMA/LABA/ICS geholfen werden.

Das zeigten die beiden Studien TRILOGY und TRINITY: Die Dreifach-Fixkombination aus extrafeinem Beclomethason-Dipropionat (100 mg), For- moterolfumarat (6 mg) und Glycopyrroniumbromid (12 mg), kurz BDP/FF/GB, beugte Exazerbationen besser vor als ICS/ LABA oder eine LAMA-Monotherapie. Die Tripeltherapie verbesserte außerdem die Lungenfunktion deutlich.

\section{Exazerbationsrisko sinkt}

In der randomisierten doppelblinden Studie TRILOGY wurde BDP/FF/GB mit BDP/FF verglichen [Lancet. 2016;88:96373]. Unter der Tripeltherapie besserte sich im Vergleich zur dualen Therapie die Lungenfunktion signifikant. Außerdem wurde das Risiko für mittelschwere und schwere Exazerbationen mit der Tripeltherapie gesenkt, und zwar um 23\% im Vergleich zur Zweierkombination.
In der TRINITY-Studie erhielten die Patienten entweder BDP/FF/GB, eine Monotherapie mit Tiotropium oder eine Tripeltherapie mit ICS/LABA plus LAMA aus zwei Inhalern. Unter beiden Tripeltherapien erlitten die Patienten im Schnitt signifikant weniger Exazerbationen als unter der Monotherapie mit Tiotropium. Die Lungenfunktion verbesserte sich. Für Prof. Dave Singh, Manchester, bietet die Tripeltherapie aus nur einem Inhaler einen großen Vorteil: „Die Therapie wird vereinfacht, was zu einer besseren Compliance der Patienten beiträgt.

\section{Ingrid Kreutz}

- Presseveranstaltung; Parma, September 2016 (Veranstalter: Chiesi)

\section{Hyperurikämie}

\section{Harnsäure: Referenzwert ist nicht gleich Zielwert}

_ Wenn ein Patient gerötete Gelenke und im Ultraschall die für eine Gicht typischen Doppelkonturzeichen hat, so muss die Diagnose Gicht lauten, auch wenn der Harnsäurewert im vom Labor genannten Referenzbereich liegt, scheinbar also normal ist, betonte Prof. Monika Reuss-Borst, Bad Kissingen. „Gicht bedeutet, dass sich Harnsäurekristalle ablagern“, erinnerte die Rheumatologin. „Harnsäure kristallisiert oberhalb des Sättigungspunkts im Gewebe aus.“ Bei Körpertemperatur und normalem $\mathrm{pH}$ Wert liege der Sättigungspunkt bei 6,5 $\mathrm{mg} / \mathrm{dl}$. Bei abnehmender Temperatur und sinkendem $\mathrm{pH}$-Wert sinkt auch der Sättigungspunkt der Harnsäure. „Bei $35^{\circ} \mathrm{Cel}$ sius liegt der Sättigungspunkt bei nur noch bei 6,0 mg/dl“, so Reuss-Borst. Das sei ein Grund, warum bei Gichtpatienten häufig das Großzehengrundgelenk be- troffen ist. Auch am Ohr zeigten sich oft Tophi („Gichtperlen“). Denn in der Peripherie ist es kälter.

"Ist die Diagnose gesichert, muss die Harnsäure unter $6 \mathrm{mg} / \mathrm{dl}$ gesenkt werden - und das langfristig“, so Reuss-Borst. Die Kristallbildung werde gehemmt, Gichtanfälle würden verhindert, Tophi lösten sich auf, und die Doppelkonturzeichen verschwänden. Die Therapie müsse mindestens über fünf Jahre erfolgen. Eine Pause sei allenfalls vertretbar, wenn der letzte Tophus verschwunden ist.

Von den beiden XanthinoxidaseHemmern Allopurinol und Febuxostat sieht Reuss-Borst letzteren im Vorteil. So würden in Deutschland über $60 \%$ der Gichtpatienten mit Allopurinol den Zielwert nicht erreichen. Mit Febuxostat $\left(\right.$ Adenuric $^{\circledR}$ ) sei eine effektivere Harnsäuresenkung möglich, es gebe weniger

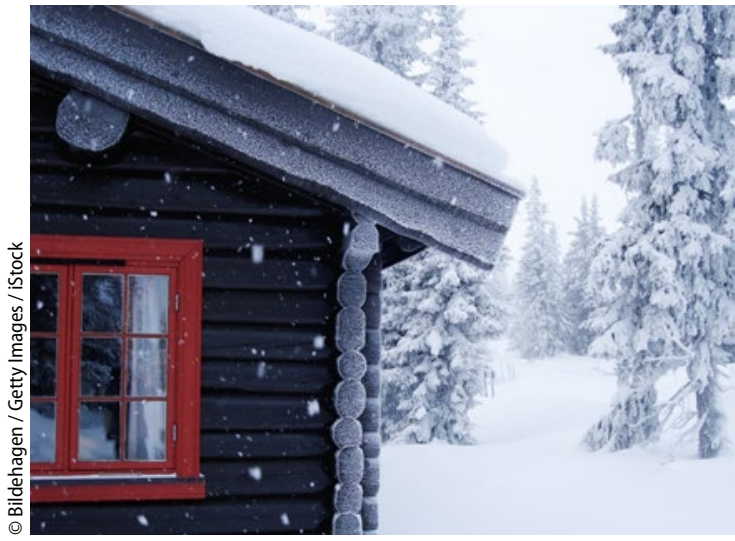

Romantischer Winter: Leider ist jetzt die Gefahr für Gichtanfälle größer.

Wechselwirkungen und es sei keine Dosisanpassung bei leichter bis mittelschwerer Nierenfunktionsstörung nötig.

Dr. Michael Hubert

- Symposium „Der therapeutische Zielwert bei symptomatischer Hyperurikämie und die Rolle der Labormedizin", 13. Jahrestagung der Deutschen Gesellschaft für klinische Chemie und Laboratoriumsmedizin; Mannheim, September 2016 (Veranstalter: Berlin-Chemie) 